\title{
Gender differences in self-perceived changes among Japanese workers with depression
}

Niki, A., Deguchi, Y., Iwasaki, S., Mitake, T., Okuda, Y., Sakaguchi, A., Hirota, T., Shirahama, Y., Nakamichi, Y., \& Inoue, K.

\begin{tabular}{|c|l|}
\hline Citation & Occupational Medicine, 70(9); 680-684 \\
\hline Issue Date & $2020-12$ \\
\hline Published & $2020-11-28$ \\
\hline Type & Journal Article \\
\hline Textversion & author \\
\hline Relation & $\begin{array}{l}\text { This is a pre-copyedited, author-produced version of an article accepted for publication } \\
\text { in Occupational Medicine following peer review. The version of record is available } \\
\text { online at: } \text { https://doi.org/10.1093/occmed/kqaa202 }\end{array}$ \\
\hline DOI & \begin{tabular}{l}
$10.1093 /$ occmed/kqaa202 \\
\hline
\end{tabular}
\end{tabular}

\section{Self-Archiving by Author(s)}

\section{Placed on: Osaka City University Repository}

Niki, A., Deguchi, Y., Iwasaki, S., Mitake, T., Okuda, Y., Sakaguchi, A., Hirota, T., Shirahama, Y., Nakamichi, Y., \& Inoue, K. (2020). Gender differences in self-perceived changes among Japanese workers with depression. Occupational Medicine, 70(9), 680-684. 
1 Gender differences in self-perceived changes among Japanese workers with

2 depression

3

$4 \quad$ Akihiro Niki $^{\dagger}$, Yasuhiko Deguchi ${ }^{\dagger *}$, Shinichi Iwasaki, Tomoe Mitake, Yukako Okuda,

5 Aya Sakaguchi, Tomoyuki Hirota, Yoshiki Shirahama, Yoko Nakamichi, Koki Inoue

6

7 Department of Neuropsychiatry, Osaka City University, Graduate School of Medicine,

8 Osaka 545-8585, Japan

9

10 Correspondence to: Yasuhiko Deguchi, Department of Neuropsychiatry, Osaka City

11 University, Graduate School of Medicine, 1-4-3, Asahi-machi, Abeno-ku, Osaka,

12 545-8585, Japan. Tel: +8166645 3821; fax: +8166636 0439; email:

13 m2012567@med.osaka-cu.ac.jp

14

15

16

17

18 
19 Abstract

20 Background: The number of patients living with depression continues to increase in

21 Japan. The economic effects of depression include loss of productivity due to both

22 absenteeism and presenteeism. Gender differences have been reported in prevalence,

23 onset pathways, and subjective symptoms of depression.

25 Aims: To understand how workers with major depressive disorder (MDD) perceive

26 problems in the workplace and examine gender differences in their self-perceived levels

27 of functioning at work, noticed during the initial stages of depression.

29 Methods: This is a cross-sectional study of Japanese workers with MDD. Participants'

30 self-perceived changes in the level of functioning at work were surveyed after the

31 diagnosis during the first visit. The relationship between gender and changes in the level

32 of functioning at work as initially perceived by the participants themselves was

33 analysed using the chi-square test, supplemented by a residual analysis.

35 Results: We administered the survey to 147 workers with MDD. In terms of gender

36 differences in initial self-perceived changes in the level of functioning at work, the 
37 proportion of men reporting reduced work efficiency was significantly higher than that

38 of women, while the proportion of women reporting deterioration in relationships with

39 colleagues and superiors was significantly higher than that of men.

40

41 Conclusions: The findings suggest that greater attention to reduced work efficiency by

42 men and to deterioration in work relationships by women with MDD should be essential

43 components of self-care. Managers need to pay attention to the level of functioning and

44 provide adequate social support for employees.

45

46 Keywords: workers, major depression disorder, change at work, self-care, social

47 support

48 
$49 \quad$ Key points

50

51 What is already known about this subject:

52 - Gender differences have been reported in the prevalence, onset pathways, and

53 subjective symptoms of MDD.

54 - Gender differences in self-perceived changes in the level of functioning at work

55 among workers with MDD remain unclear.

56

57 What this study adds:

- There were significant gender differences in self-perceived changes in the level of

59 functioning at work among workers with MDD.

60

61 What impact this may have on practice or policy:

- Male and female workers should pay attention to reduced work efficiency and deterioration in work relationships as part of self-care; managers should pay attention to

64 the level of functioning in their employees to provide appropriate social support.

65

66 


\section{Introduction}

68 Nearly 163 million people worldwide were affected by major depressive disorder

69 (MDD), an important cause of disability in young and middle-aged adults, in 2017 [1]

70 (refer to 'Mental disorders' section of Table1). The economic effects of depression

71 include productivity loss due to both absenteeism and presenteeism [2,3]. A recent study

72 of European workers estimated the cost of productivity loss from depression to be $€ 77$

73 billion per year [4]. In addition to malignancies, acute myocardial infarction, stroke and

74 diabetes, mental illnesses have been among the five major diseases in Japan since 2011.

75 To ensure that adequate mental health care is provided, a review of the medical system

76 is required. In 2017, patient surveys by the Ministry of Health, Labour and Welfare in

77 Japan reported that the number of patients with mood disorders, including MDD, had

78 increased from about 0.9 million to about 1.2 million in the last 15 years, and that the

79 number of patients aged under 65 years with mood disorders continues to increase [5].

80 In Japan, the mandatory retirement age is generally over 60 , and most people under the

81 age of 65 are expected to have sufficient work ability to work full-time.

82 It is necessary to recognize the fact that anyone can suffer from depression, as this can

83 help in early detection of the disorder and appropriate treatment. Failure to diagnose

84 MDD at an early stage may result in significant losses to patients, their families, 
colleagues and employers. It is well known that individuals with MDD have a high risk

86 of suicide [6]; improved mental health care is therefore important to prevent suicide. In

87 this context, as a form of mental health care, self-care is an important approach to the management of long-term health and the prevention of ill health by living a healthy

89 lifestyle [7]. The World Health Organization defines self-care as 'the ability of

90 individuals, families and communities to promote, maintain health, prevent disease and

91 to cope with illness with or without the support of a health care provider' [8]. Moreover,

92 early detection of MDD either by workers themselves, colleagues, superiors or

93 healthcare providers is also important to prevent economic loss to the country as a

94 whole.

95 However, many patients with MDD often lack awareness of their illness and have

96 difficulty seeking help during the early stages. Early symptoms of MDD not only

97 include medical symptoms such as depressed mood or insomnia, but also occupational

98 problems such as inability to perform normal work or absence from work $[9,10]$.

99 Colleagues and supervisors at work are usually not medical or mental health

100 professionals and are unable to accurately detect medical symptoms of depression in

101 workers. Therefore, understanding changes in mood, level of functioning, performance,

102 etc., early by individuals with MDD at work and by colleagues and supervisors is 
essential to improve countermeasures for MDD in the workplace. However, few

104 previous studies have focused on initial changes in MDD patients observed at work.

105 In general, the prevalence of depression in women is about twice higher than in men,

106 and gender differences in the onset pathways have been reported [11]. Blatt suggests

107 that there are two categories of depression: anaclitic depression, which is characterized

108 by a vulnerability to interpersonal relationships and more common among women; and

109 introjective depression, which is characterized by a vulnerability to self-esteem and is

110 more common among men [12]. Furthermore, there are gender differences in subjective

111 symptoms of depression, and it has been reported that women are more likely than men

112 to be aware of their increased appetite, weight gain, anger, etc. [13]. We have previously

113 emphasized the significance of focusing on gender differences when studying the

114 relationship between perceived individual-level occupational stress and hazardous

115 alcohol consumption among Japanese teachers [14]. In the present study, we

116 hypothesised that there may be gender differences in the initial self-perceived changes

117 in the level of functioning at work in individuals with depression.

118 We aimed to identify and understand the initial self-perceived changes in the level of

119 functioning at work among workers with MDD, and the gender differences in these changes. To our knowledge, this is the first study investigating gender differences in 
121 self-perceived changes in the level of functioning at work among workers with MDD.

\section{Methods}

124 A total of 147 outpatients with MDD participated in this cross-sectional study. The 125 study was conducted from March 2011 to March 2019, and all participants received 126 treatment at the Department of Neuropsychiatry, Osaka City University Hospital, Osaka, 127 Japan. All the participants were over the age of 20 and were working or on sick leave at 128 the first visit.

129 MDD was diagnosed by multiple psychiatrists with over five years of training using 130 the Mini International Neuropsychiatric Interview (MINI) for diagnosing mental illness;

131 the International Classification of Diseases-10 [15]; and the Diagnostic and Statistical

132 Manual, $4^{\text {th }}$ Edition, Text Revision (DSM-IV-TR) criteria [16]. The MINI is a brief, 133 structured interview developed by general clinicians and psychiatrists in Europe and the

134 United States; its reliability and validity have been established $[17,18]$. We used the

135 MINI module for the diagnosis of an MDD episode. Individuals with a primary 136 diagnosis of schizophrenia, anxiety disorders other than MDD, other substance use 137 disorders, intellectual disability and major medical disorders including neurological 138 disorders, pregnancy, and serious suicide attempts were excluded. 
139 The attending physician explained the study purpose and methods to the participants,

140 all the participants provided informed consent to voluntarily participate, and understood

141 that there was no penalty for choosing not to participate. After a sufficient explanation

142 of the study for participants had been provided, written informed consent was obtained

143 from each participant. Patient records/information were anonymised and de-identified

144 prior to analysis. Data from 147 participants who agreed to participate in this study were

145 analysed. The study was approved by the Human Subjects Review Committee at Osaka

146 City University (approval number: 4245).

147 To maintain and promote mental health care in workers, the Ministry of Health,

148 Labour and Welfare has called on supervisors in Japan to pay attention to changes in

149 workers' levels of functioning at work such as reduced work efficiency, late arrival,

150 absence from work, early departure, etc. Based on this guideline, we selected the

151 following indicators: 'late arrival, absence from work, early departure', 'reduced work

152 efficiency', 'deterioration in relationships with colleagues and superiors', 'increase in

153 smoking and drinking rates' and 'other' [19]. In accordance with the above,

154 self-perceived changes in the level of functioning of participants at work were surveyed

155 after the diagnosis at the first visit. Participants were asked to choose the levels of

156 functioning they thought applied to them. Data on demographic and occupational 
157 variables of the participants were also collected, including age, sex, job category,

158 position classification, and type of employment. The Hamilton Depression Rating Scale

159 (HAM-D) was used to assess the severity of depression at the initial visit.

160 Statistical analyses were conducted using SPSS statistical version 25 (IBM Corp,

161 Armonk, NY, USA). Sociodemographic data on participants were examined using

162 descriptive statistics. The relationship between self-reported initial changes in the level

163 of functioning at work and gender was analysed using the chi-square test based on

164 Cochrane's criteria, supplemented by a residual analysis (significance level set at $P<$ $1650.05)[20]$.

166

167 Results

168 The demographic and occupational characteristics of the participants are summarised in

169 Table 1. Of the 147 participants, 101 were men (69\%) and 46 were women (31\%). The

170 mean age was 39.8 years (standard deviation $[\mathrm{SD}]=10.2$ ). There were 82 professionals

171 (56\%), 34 clerical workers (23\%) and 31 hospitality workers (21\%); 54 managerial

172 employees (37\%) and 93 non-managerial employees (63\%). In terms of type of

173 employment, $128(87 \%)$ were regular employees and $19(13 \%)$ temporary employees.

174 The mean HAM-D score was $21.0(\mathrm{SD}=8.3)$, and there were no significant gender 
differences in HAM-D scores.

176 Initial self-reported changes in the level of functioning at work by gender are

177 summarised in Table 2. The most common change in the level of functioning at work as

178 perceived by the participants themselves was 'reduced work efficiency', followed by

179 'late arrival, absence from work, early departure'. By gender, the most common change

180 in the level of functioning at work that male participants initially perceived was

181 'reduced work efficiency', followed by 'late arrival, absence from work, early

182 departure', while for female participants, it was 'reduced work efficiency', followed by

183 'deterioration in relationships with colleagues and superiors'. The chi-square test

184 showed that initial self-reported changes in the level of functioning at work were

185 significantly associated with gender. In addition, residual analysis showed that the

186 proportion of men reporting 'reduced work efficiency' was significantly higher than that

187 of women, while the proportion of women reporting 'deterioration in relationships with

188 colleagues and superiors' was significantly higher than that of men. The answers of the

189 eleven participants who chose 'other' were 'increased time spent alone' for seven,

190 'headache' for one, and unknown for the other three.

191

192 Discussion 
193 In this study, we investigated self-perceived changes in the level of functioning at work

194 in the initial stages of depression among workers with MDD, and gender differences in

195 these changes. Our results show that the most common self-perceived change in the

196 level of functioning at work in workers with MDD was 'reduced work efficiency': the

197 proportion of males reporting 'reduced work efficiency' was significantly higher than

198 that of females and the proportion of females reporting 'deterioration in relationships

199 with colleagues and superiors' was significantly higher than that of males.

200 The strength of this study is that this is the first study to our knowledge to investigate

201 gender differences in self-perceived changes in the level of functioning at work among

202 workers with MDD. Our results suggest that gender differences should be taken into

203 account to ensure early detection of depression among workers. The limitations of this

204 study are as follows. First, the sample size was small. Second, although the prevalence

205 of depression was almost twice as high in women [21], there were more male than

206 female participants; therefore, the sex ratio was biased. Third, the study included items

207 about past memory, and recall bias may have influenced participant responses. Fourth,

208 as this was a single-centre study, the study sample has limited representativeness. Future

209 multicentre studies should be conducted for further research into the early detection of

210 MDD in the workplace. Fifth, it was considered that there were differences in the way 
211 of working between male and female workers based on differences in position

212 classification, and the impact of position classification on self-reported initial changes

213 in the level of functioning at work should be adjusted for. However, we could not

214 evaluate the effect of position classification for these outcomes due to the lack of

215 statistical power. They should be focused on in future studies.

216 Results of Kendler et al.'s study on gender differences in the pathogenesis of

217 depression among 208 pairs of heterosexual dizygotic twins in whom only one of the

218 twins developed depression suggest that parental warmth, neuroticism, divorce, social

219 support and marital satisfaction had a greater impact in women as risk factors for

220 developing depression. In contrast, childhood sexual abuse, conduct disorder, drug

221 abuse, prior history of major depression, and distal and dependent proximal stressful life

222 events had a greater impact in men as risk factors for developing depression. In men,

223 financial, occupational and legal categories of life events had a stronger effect on the

224 development of depression [11]. Blatt suggests that depression can be divided into two

225 types: anaclitic depression and introjective depression. The former arises from

226 interpersonal problems and tends to be more prevalent among women, while the latter

227 arises from stressful events affecting self-esteem such as academic failure, and tends to

228 be more prevalent among males. He attributes this to the fact that women are more 
229 likely than men to interact with same-sex friends and think more about their

230 relationships [12].

231 Our study found that 'reduced work efficiency' was reported significantly more often

232 by men than be women, while women were more significantly more likely than men to

233 perceive a 'deterioration in relationships with colleagues and superiors'. The results of

234 the Kendler et al. study and the analytical study by Blatt suggest that men are more

235 sensitive to failure to achieve goals and women more sensitive to interpersonal

236 relationships, which may also help explain our results. Failure to achieve goals might be

237 interpreted as 'reduced work efficiency' after the onset of depression in men, while

238 women may perceive vulnerability in interpersonal relationships as a 'deterioration in

239 relationships with colleagues and superiors' after the onset of depression.

240 Martin et al. analysed data from a cohort of 265 patients with mild depression who

241 reported current employment to show that even subclinical depression has been reported

242 to reduce performance at work [22]. Beck et al. analysed data from 771 patients with

243 depression with current employment to highlight the relationship between minor

244 symptoms of depression and productivity loss [23]. These explain the fact that the most

245 common early changes in the level of functioning at work due to MDD among both men

246 and women in this study was reduced work efficiency. This finding may be consistent 
247 with the main signs and symptoms of a depressive episode, such as having low

248 motivation, poor concentration, cognitive functioning decline, and difficulty in making

249 decisions.

250 It has also been reported that women with depression were more likely than men to

251 express anger or hostility toward others, which may lead to a significantly higher

252 percentage of women with MDD reporting 'deterioration in relationships with

253 colleagues and superiors' than men [13].

254 Enhanced self-care and social support are essential for the early detection of MDD.

255 Our results suggest that as self-care measures, workers, especially males, should pay

256 greater attention to their reduced work efficiency, and female workers should pay more

257 attention to the deterioration in their relationships with colleagues and superiors.

258 Managers are in a position to lead workers to seek further advice from their health care

259 providers by noticing reduced work efficiency in male and female workers and the

260 deterioration of relationships with colleagues and superiors among female workers and

261 by subsequently having heart-to-heart conversations with them. Therefore, managers are

262 required to acquire and improve their communication skills. By focusing on changes in

263 workers' performance and interactions at the workplace, early detection of MDD and

264 therefore, early treatment may be possible. 
266 Funding

267 None declared.

268

269 Acknowledgements

270 We would like to thank all participants for their contributions.

271

272 Competing interests

273 None declared.

274

275

276

References

277

1. GBD 2017 Disease and Injury Incidence and Prevalence Collaborators. Global,

regional, and national incidence, prevalence, and years lived with disability for 354

279

diseases and injuries for 195 countries and territories, 1990-2017: a systematic

280 analysis for the Global Burden of Disease Study 2017. Lancet 2018; 392(10159): $1789-1858$.

2. Kessler RC, Akiskal HS, Ames M, Birnbaum H, Greenberg P, Hirschfeld RMA, et al. Prevalence and effects of mood disorders on work performance in a nationally representative sample of U.S. workers. Am J Psychiatry 2006; 163(9): 1561-1568.

3. Knapp M. Global patterns of workplace productivity for people with depression: 
absenteeism and presenteeism costs across eight diverse countries. Soc Psychiatry Psychiatr Epidemiol 2016; 51(11): 1525-1537.

4. Evans-Lacko S, Knapp M. Importance of social and cultural factors for attitudes, disclosure and time off work for depression: Findings from a seven country european study on depression in the workplace. PLoS One 2014; 9(3): e91053.

5. Ministry of Health, Labour and Welfare. Patient Survey 2017. [Internet]. Tokyo: MHLW, 2017. Available from: https: //www.mhlw.go.jp/toukei/saikin/hw/kanja/17/index.html [Japanese] Accessed May 2020.

6. Isometsä E. Suicidal behaviour in mood disorders-who, when, and why? Can J Psychiatry 2014; 59(3): 120-130.

7. Lucock M, Gillard S, Adams K, Simons L, White R, Edwards C. Self-care in mental health services: A narrative review. Heal. Soc. Care Community 2011; 19(6): $602-16$

8. World Health Organization, Regional Office for South-East Asia. Self-care for heart health. [Internet]. 2014. Available from: https://apps.who.int/iris/handle/10665/205887 Accessed May 2020. 
304 Insomnia as a predictor of mental disorders: A systematic review and meta-analysis.

$305 \quad$ Sleep Med Rev 2019; 43(November): 96-105.

306 10. Johnston DA, Harvey SB, Glozier N, Calvo RA, Christensen H, Deady M. The

307 relationship between depression symptoms, absenteeism and presenteeism. J Affect

308 Disord 2019; 256(June): 536-540. Available from: https:

$309 \quad$ //doi.org/10.1016/j.jad.2019.06.041 Accessed May 2020.

310 11. Kendler KS, Gardner CO. Sex differences in the pathways to major depression: a

311 study of opposite-sex twin pairs. Am J Psychiatry 2015; 171(4): 426-435.

312 12. Blatt S. Experiences of depression: theoretical, clinical, and research perspectives.

313 Washington, DC: American Psychological Association, 2004.

314 13. Frank E, Carpenter LL, Kupfer DJ. Sex differences in recurrent depression: are there

$315 \quad$ any that are significant? Am J Psychiatry 1988; 145(1): 41-45.

316 14. Deguchi Y, Iwasaki S, Kanchika M, Nitta T, Mitake T, Nogi Y, et al. Gender

317 differences in the relationships between perceived individual-level occupational

318 stress and hazardous alcohol consumption among Japanese teachers: A

319 cross-sectional study. PLoS One 2018; 13(9): e0204248.

320 15. World Health Organization. ICD-10: International statistical classification of

321 diseases and related health problems, 10th Revision. Geneva, Switzerland: WHO; 
16. Association Psychiatric Association. Diagnostic and statistical manual of mental disorder, 4th Edition. Washington, DC: American Psychiatric Association, 2000.

17. Otsubo T, Tanaka K, Koda R, Shinoda J, Sano N, Tanaka S, et al. Reliability and 1954; 10: 417-451. 
340 21. Albert PR. Why is depression more prevalent in women? J Psychiatry Neurosci

$341 \quad 2015 ; 40(4): 219-221$.

342 22. Martin JK, Blum TC, Beach SR, Roman PM. Subclinical depression and

343 performance at work. Psychiatry Psychiatr Epidemiol 1996; (31): 3-9.

344 23. Beck A, Crain AL, Solberg LI, Glasgow RE, Maciosek MV. Severity of depression

345 and magnitude of productivity loss. Ann Fam Med 2011; (9): 305-311.

346 
347 Table 1. Demographic and occupational characteristics $(n=147)$.

\begin{tabular}{|c|c|c|c|c|}
\hline & Men & Women & $t$-test & chi-square test \\
\hline & $(n=101)$ & $(n=46)$ & & \\
\hline & $n(\%)$ & $n(\%)$ & & \\
\hline Age, years, mean $( \pm \mathrm{SD})$ & $40.4( \pm 10.1)$ & $38.6( \pm 10.3)$ & NS & \\
\hline \multicolumn{5}{|l|}{ Job category } \\
\hline Professionals & $51(50 \%)$ & $31(67 \%)$ & & NS \\
\hline Clerical workers & $25(25 \%)$ & $9(20 \%)$ & & \\
\hline Hospitality workers & $25(25 \%)$ & $6(13 \%)$ & & \\
\hline \multicolumn{5}{|l|}{ Position classification } \\
\hline Non-managerial & $57(56 \%)$ & $36(78 \%)$ & & $* *$ \\
\hline Managerial & $44(44 \%)$ & $10(22 \%)$ & & \\
\hline \multicolumn{5}{|l|}{ Type of employment } \\
\hline Regular & $91(90 \%)$ & $37(80 \%)$ & & NS \\
\hline Temporary & $10(10 \%)$ & $9(20 \%)$ & & \\
\hline HAM-D score, mean $( \pm$ SD) & $21.9( \pm 8.5)$ & $19.0( \pm 7.4)$ & NS & \\
\hline
\end{tabular}

348 SD: standard deviation; HAM-D: Hamilton Depression Rating Scale; NS: not

349 significant, ${ }^{* *} P<0.01$ 
Table 2. Gender differences in self-perceived initial changes in the level of functioning at work

\begin{tabular}{|c|c|c|c|c|c|}
\hline & \multirow{2}{*}{$\begin{array}{c}\text { Men } \\
(n=101) \\
n(\%)\end{array}$} & \multirow{2}{*}{$\begin{array}{l}\text { Women } \\
(n=46) \\
n(\%)\end{array}$} & \multirow[t]{2}{*}{ chi-square test } & \multicolumn{2}{|c|}{ Adjusted residual } \\
\hline & & & & Men & Women \\
\hline Reduced work efficiency & $54(53 \%)$ & $16(35 \%)$ & * & 2.1 & -2.1 \\
\hline Late arrival, absence from work, early departure & $21(21 \%)$ & $6(13 \%)$ & NS & 1.1 & -1.1 \\
\hline Deterioration of relationships with colleagues and superiors & $9(9 \%)$ & $13(28 \%)$ & $* *$ & -3.0 & 3.0 \\
\hline Increase in smoking and drinking rates & $12(12 \%)$ & $5(11 \%)$ & NS & 0.2 & -0.2 \\
\hline Other & $5(5 \%)$ & $6(13 \%)$ & NS & -1.7 & 1.7 \\
\hline
\end{tabular}

NS: not significant, ${ }^{*} P<0.05,{ }^{* *} P<0.01$ 